\title{
Estado del arte de la investigación universitaria en Desarrollo Económico Local en Colombia
}

\author{
Álvaro Albán Moreno" / Jorge Alberto Rendón Vélez *"
}

\section{Resumen}

Este artículo presenta el estado del arte de la investigación universitaria en Desarrollo Económico Local en Colombia. El diseño metodológico se centró en un proceso hermenéutico y crítico de tres fases: fase descriptiva, fase interpretativa y fase de valoración crítica. Por lo tanto fue necesario desarrollar diferentes instrumentos para obtener la información de los productos de investigación de los grupos reconocidos por Colciencias. Los resultados permiten categorizar los problemas de investigación, las tendencias teóricas sobre el desarrollo, los enfoques metodológicos, los resultados y conclusiones; y finalmente, reconstruir críticamente dichos resultados y aportar algunas recomendaciones. Se destacan, explicita o implícitamente, los enfoques teóricos integrales sobre el desarrollo, tanto en general, como cuando se utiliza la acepción local; y el avance de perspectivas cualitativas en los diseños investigativos. La multidimensionalidad, transdisciplinariedad, holismo, integralidad y el carácter de estructura del Desarrollo Económico Local son significados que ganan espacio en la investigación reciente sobre el tema.

Palabras clave: economía, desarrollo local, multidimensionalidad, estructura, sistema, holismo, localidad.

\section{State of the art in University RESEARCH ABOUt Local Economic Development in Colombia Abstract}

This article presents the state of art in university research about Local Economic Development in Colombia. The methodological design was based on a hermeneutic and critical process divided in three phases: descriptive, interpretative and, evaluative. Therefore, it was necessary to design several instruments to get the information from researching products of investigation groups recognized by Colciencias. The results help to categorize researching problems, theoretical tendencies about development, methodological approaches, results and conclusions, and reconstruct them to propose some recommendations. Comprehensive theoretical approaches about development both in general sense and in local sense and the advancement of qualitative approaches in research designs are implicitly or explicitly emphasized. Multidimensionality, transdisciplinar approach, holism, integrality and the sense of structure of Economic Local Development are new concepts that have gained relevance in the recent research of this subject.

Key words: Economy, local development, Multidimensionality, structure, system, holism, locality.

* Docente tiempo completo e investigador Centro de Investigaciones Económicas del Programa de Economía de la Universidad Libre Seccional Cali. Líder Grupo de Investigación en Desarrollo Local y Regional, Colciencias 0015769. Correo electrónico: alvaro.alban@email.unilibrecali.edu.co

* Docente tiempo completo e investigador Centro de Investigaciones Económicas del programa de Economia de la Universidad Libre Seccional Cali. Miembro Grupo de Investigación en Desarrollo Local y Regional. Colciencias, Colciencias 0015769.

Correo electrónico: jorge.rendon@email.unilibrecali.edu.co

Fecha de recepción: diciembre 11 de 2007.

Fecha de aprobación: febrero 8 de 2008. 


\section{INTRODUCcIón}

El Desarrollo Económico Local (DEL) constituye en la actualidad un tema de amplia reflexión académica. En él confluyen prácticas, teorías, y diferentes acepciones para nominarlo, entre las que se destacan: proceso, instrumento, estrategia, actividad e iniciativa de política.

La literatura sobre $D E L$, incluye una variedad de posibilidades. Una de ellas está constituida por el análisis de las condiciones y condicionantes que lo promocionan o inhiben: innovación y tecnología, descentralización, solidaridad y cooperación, institucionalidad, territorio y capital humano, entre otros. En el DEL confluyen diferentes niveles de actuación (políticoadministrativo, cultural-institucional, geográfico-ambiental y económico-productivo) y áreas de estudio e investigación (Economía y Desarrollo Regional, Geografía económica, Economía Urbana y Distritos industriales y Desarrollo Económico de las Comunidades). De igual forma, hay múltiples factores -tangibles e intangibles-, que lo afectan. También intervienen en él diversos agentes públicos y privados (Cuervo, 2005 y Tello, 2006). En este orden de ideas, los propósitos del $D E L$, abarcan lo económico (competitividad, mercados, productividad y eficiencia), lo sociocultural (redes sociales, participación, empoderamiento social, pertenencia y valores) y lo político (descentralización, democratización y participación).

Con esta investigación se busca avanzar hacia una mayor y mejor comprensión de las acciones que son dirigidas por diferentes agentes desde y hacia las localidades con miras a impulsar su bienestar. Se pretende entonces, que los agentes que participan en procesos $D E L$-investigadores, académicos, funcionarios públicos, administradores locales, empresarios y líderes locales- cuenten con información sobre las diferentes prácticas y sustentos teóricos que caracterizan y enmarcan estos procesos.
El punto de partida de este estudio lo constituye el interés por ampliar la comprensión sobre los procesos locales que pretenden la consecución del bienestar de las poblaciones, como perspectiva posible de desarrollo económico. La insuficiencia de este entendimiento, conlleva seguramente a la obtención de resultados limitados por parte de los diseñadores de los programas de intervención y demás agentes involucrados. Las evidencias indican que a la fecha no hay un trabajo investigativo que sistematice y valore lo alcanzado hasta ahora por las investigaciones universitarias sobre Desarrollo Económico Local en Colombia.

En el contexto nacional, los esfuerzos por encontrar solución a los principales problemas en el ámbito local y desde lo local, como propósitos de desarrollo económico, constituyen un importante reto académico para disponer de un conocimiento en el que mandatarios, académicos y comunidades encuentren la oportunidad de reconocerse y avanzar en el perfeccionamiento de planes de trabajo a partir de la identificación, sistematización, reconstrucción, valoración de la apropiación social y otras posibilidades, de los diversos enfoques conceptuales, modelos y estrategias metodológicas aplicadas en investigaciones en desarrollo local. Este reconocimiento, se presume, debe conllevar a una mejoría en los resultados de las intervenciones y por tanto al favorecimiento de la comunidad.

En la visión teórica del proyecto se asumió al $D E L$ como un concepto integrador (holístico) de todas las dimensiones de la vida social de una comunidad, que además de dar cuenta de aspectos específicamente de naturaleza económica, establece su relacionamiento e impacto en las esferas política y cultural (Cavestany, 2000; Alburquerque, 2004; Tello, 2005).

Se define como referente conceptual, que se consideran investigaciones en $D E L$, aquellas que indagan sobre alguna o algunas dimensiones específicas del 
desarrollo económico y su conexión con los aspectos culturales y políticos. Estos tres aspectos, en sentido amplio, abarcan la dinámica social de una comu- nidad; además, se asume, que el proceso implica la participación de la comunidad. El Cuadro 1 detalla esta relación.

\section{Cuadro 1. Dimensiones económicas y sus relaciones}

\begin{tabular}{|l|l|}
\hline \multicolumn{1}{|c|}{ DIMENSIONES ECONÓMICAS } & \multicolumn{1}{|c|}{ RELACIONES POLÍTICAS Y CULTURALES } \\
\hline - Aumento producción local & \\
- Mercado de trabajo: cantidad y & - Políticas públicas articuladas a la experiencia \\
calidad del empleo generado. & investigativa \\
- Desarrollo cadenas productivas & Movilización actores sociales \\
- Sustentabilidad de proyectos a & - Mecanismos de institucionalización \\
largo plazo & de los procesos \\
- Ampliación de la competitividad & Correlación de los procesos con \\
sistémica local & los factores condicionantes \\
- Mejoramiento del nivel de renta & - Reflejos de las experiencias con las relaciones \\
- Infraestructura social & de poder de la comunidad. \\
local - Inversión pública & Compromiso del sector público y actuación \\
- Planeación del desarrollo & del gobierno local y otros niveles \\
\multicolumn{2}{|l}{ - Generación capital social } \\
\hline
\end{tabular}

Fuente: elaboración propia con base en Cavestany (2000), Alburquerque (2004) y Tello (2005).

Aunque se parte de la perspectiva que el $D E L$ es un proceso de naturaleza participativa y dialógica, esta investigación consultó los procesos organizativos, evaluativos y de gestión social en los que participe o no directamente la comunidad o sector social implicado, son asumidos por los investigadores con la calificación de "local".

El objetivo general se centra en la apropiación crítica y en hacer aportes valorativos, sobre el conocimiento acumulado en la investigación universitaria sobre DEL. Para el efecto, se identificó la naturaleza de los problemas formulados, las tendencias teóricas y metodológicas implícitas o explícitas, resultados, conclusiones y los elementos políticos y culturales asociados a las investigaciones; además se reconstruyó interpretativamente el conocimiento generado por las investigaciones en el contexto colombiano. Finalmente se valora y presentan recomendaciones sobre los resultaos de dichos estudios. La discusión, se consigna en la medida que se presentan los resultados. En las conclusiones se presentan los interrogantes que sugiere el presente trabajo y la aproximación a su respuesta. El Cuadro 2, resume el problema de investigación y los objetivos del proyecto.

\section{Método}

El diseño metodológico se centró en un proceso hermenéutico y crítico en tres fases: descriptiva, interpretativa y valoración crítica.

El trabajo requirió un proceso de revisión documentaria, el desarrollo de instrumentos de clasificación, categorización y sistematización y, finalmente, un proceso de discusión conceptual alrededor de los resultados obtenidos, que fue sistematizado en documentos denominados anexos. 


\section{Cuadro 2. Problema y objetivos de investigación}

\begin{tabular}{|c|c|c|}
\hline PROBLEMA & OBJETIVO GENERAL & OBJETIVOS ESPECÍFICOS \\
\hline $\begin{array}{l}\text { ¿Cuáles es la naturaleza } \\
\text { de los problemas formu- } \\
\text { lados, conclusiones y los } \\
\text { elementos políticos y cul- } \\
\text { turales asociados a la in- } \\
\text { vestigación universitaria } \\
\text { en desarrollo económico } \\
\text { local en Colombia? }\end{array}$ & $\begin{array}{l}\text { Apropiar críticamente y } \\
\text { hacer aportes valorativos, } \\
\text { sobre el conocimiento } \\
\text { acumulado en la inves- } \\
\text { tigación universitaria en } \\
\text { desarrollo económico lo- } \\
\text { cal en Colombia }\end{array}$ & $\begin{array}{l}\text { - Identificar la naturaleza de los problemas } \\
\text { formulados, las tendencias teóricas y } \\
\text { metodológicas implícitas o explícitas, } \\
\text { resultados y conclusiones en la } \\
\text { investigación universitaria en desarrollo } \\
\text { económico local en Colombia. } \\
\text { - Sistematizar los elementos señalados en el } \\
\text { objetivo específico número uno. } \\
\text { - Reconstruir interpretativamente el } \\
\text { conocimiento generado por la investigación } \\
\text { en desarrollo económico local en el } \\
\text { contexto colombiano. } \\
\text { - Formular valoraciones y recomendaciones } \\
\text { sobre los resultados y conclusiones de la } \\
\text { investigación universitaria en desarrollo } \\
\text { económico local en Colombia, desde una } \\
\text { perspectiva que involucre las dimensiones } \\
\text { política y cultural de las comunidades que } \\
\text { han desarrollado y ejecutado este tipo de } \\
\text { proyectos investigativos. }\end{array}$ \\
\hline
\end{tabular}

Fuente: protocolo de investigación.

Como unidad de análisis se tomaron los productos -artículos científicos, tesis de maestría, libros y capítulos de libro- de las investigaciones sobre $D E L$ elaborados por los Grupos de Investigación Universitaria inscritos y reconocidos por Colciencias. Se identificaron 380 grupos registrados y reconocidos a nivel nacional que, de acuerdo con la clasificación temática correspondiente, han reportado proyectos de investigación asociados al tema del Desarrollo Económico Local en Colombia. Esta información fue depurada posteriormente mediante una revisión de las líneas de investigación declaradas por los grupos y las temáticas de los respectivos productos, lo que permitió establecer que solamente 46 de los grupos señalados anteriormente realizaron estudios relacionados con el tema de investigación o con áreas afines tales como Desarrollo Económico, Desarrollo Social, Desarrollo Regional y Descentralización Política y Administrativa, entre otras.

Dado que el presente estudio centraba su atención específicamente en las investigaciones que tratan el tema del Desarrollo Económico Local en Colombia, en relación con los elementos económicos y productivos y desde una perspectiva local (proceso de decisiones de corte participativo que implican el uso de recursos y factores locales), ${ }^{1}$ posteriormente fue necesario aplicar un nuevo filtro de selección cuyo resultado fue la inclusión de solamente 19 grupos en el estudio, sobre los cuales se procedió posteriormente a realizar una selección de 85 productos, según su pertinencia para el tema estudiado. Sin embargo,

1 Tres características fueron evaluadas fundamentalmente para establecer la pertinencia de los productos para el desarrollo del estudio: a) que la investigación se relacionara con temas como bienestar, calidad de vida o contemplara implícitamente o explícitamente el concepto de desarrollo local, b) que en dichos temas se incluyeran elementos de naturaleza económica o productiva y c) que las investigaciones evidenciaran el uso de factores y recursos locales o la participación de la comunidad en los procesos abordados respecto a aspectos tales como la toma de decisiones, la definición de metas u objetivos de bienestar, calidad de vida y desarrollo, así como en la configuración y solución de los problemas estudiados. 
una posterior revisión temática, permitió establecer que, de acuerdo con el criterio del grupo de investigadores, solamente 29 productos reunían los requisitos de adecuación temática para el presente trabajo, siendo estos los productos finalmente analizados. ${ }^{2}$

Resultados

\section{Naturaleza de los PROBlemas abordados POR LAS INVESTIGACIONES EN DEL}

El Cuadro 3 presenta la clasificación de la naturaleza de los problemas abordados de acuerdo con las ca- tegorías establecidas por el grupo de investigación. Es importante señalar que algunas investigaciones pueden relacionarse con dos o más de las categorías establecidas, situación que se hace evidente en los resultados presentados en la tabla. Diez y ocho de los productos de investigación abordados (62\%) indagan sobre la existencia de diversos tipos de relaciones teóricas, para lo cual se analizan las conexiones entre ciertos elementos de naturaleza socioeconómica y conceptos como el de calidad de vida, desarrollo y bienestar, entre otros.

Cuadro 3. Naturaleza de los problemas abordados

\begin{tabular}{|l|c|}
\hline \multicolumn{1}{|c|}{ Tipo de problema abordado } & No de investigaciones \\
\cline { 2 - 3 } & \multicolumn{1}{|c|}{ Establecimiento de relaciones conceptuales o teóricas } \\
\hline Exploración teórica & 8 \\
\hline Comprobación de hipótesis & 5 \\
\hline Elaboración de instrumentos analíticos & 2 \\
\hline $\begin{array}{l}\text { Contrastación o comprobación empírica de un modelo, } \\
\text { o de estrategias o metodologías DEL }\end{array}$ & 1 \\
\hline $\begin{array}{l}\text { Evaluación de la aplicación de un instrumento, una es- } \\
\text { trategia (pública o privada) o una metodología }\end{array}$ & 6 \\
\hline Resolución de un problema empírico & 2 \\
\hline Construcción de procesos comunitarios & 2 \\
\hline Sistematización de una experiencia comunitaria & 17 \\
\hline
\end{tabular}

Fuente: Anexo 4 investigación - Síntesis Productos Descritos.

Las relaciones conceptuales o teóricas señaladas en el ítem primero, alrededor de las cuales giran las preguntas e hipótesis de las investigaciones, son:
- Capital social y su relación con calidad de vida y el desarrollo local.

- Gobernabilidad, participación y desarrollo.

2 Con el fin de clasificar y sistematizar la información obtenida de los productos, se utilizaron diferentes instrumentos. El Anexo 1 (Inventario), permitió identificar y clasificar los diversos tipos de productos de investigación asociados al tema DEL, a partir de las instituciones universitarias incluidas en la base de datos del Colciencias. El Anexo 2 (Preselección) facilitó seleccionar y depurar los productos inicialmente identificados, es decir, verificar su pertinencia temática. Para tal efecto la ficha capturó fundamentalmente información sobre el tema objeto de investigación, palabras y conceptos claves y una breve síntesis del texto. Mediante el Anexo 3 (Descripción Productos), se describió la información obtenida (fase descriptiva) de los productos finalmente seleccionados: autor, clase de producto, lugar y referencia bibliográfica de la publicación, grupo de investigación que realizó el trabajo, Institución a la que pertenece el grupo de investigación, problema formulado y/o hipótesis, elementos teóricos implícitos o explícitos alrededor de conceptos como desarrollo, desarrollo local y desarrollo económico local, características metodológicas del estudio y, finalmente, resultados y conclusiones de la investigación. El Anexo 4 (Síntesis Productos Descritos) se elaboró como un instrumento que permitiera, registrar, clasificar, categorizar y sistematizar la información, con el propósito de sintetizar los contenidos conceptuales que emergieron en la fase interpretativa (Anexo 3). En este instrumento se abordaron procesos como la clasificación y el registro en categorías de aspectos tales como los problemas formulados y sus hipótesis, las posibles orientaciones conceptuales o teóricas sobre el desarrollo en general y particularmente sobre el $D E L$, los enfoques metodológicos detectados en las investigaciones y los aspectos fundamentales de las conclusiones o valoraciones realizadas por los autores de los productos. 
- Dinámica sectorial, gestión fiscal y desarrollo.

- Motivación hacia el trabajo comunitario y desarrollo humano.

- Formas de liderazgo y desarrollo comunitario.

- Competitividad, localidad y desarrollo.

- Cultura y pobreza.

- Métodos participativos y economía experimental.

- Relaciones productivas y de generación de excedentes; y calidad de vida.

- Calidad de vida y percepciones subjetivas de capacidades y expectativas.

- Información y decisiones sobre desarrollo comunitario y local.

- Educación, cultura y desarrollo local.

- Participación comunitaria y desarrollo.

- Desarrollo de habilidades de comunicación y liderazgo en el núcleo familiar y desarrollo comunitario.

- Conflicto, territorio y desarrollo regional.

Las relaciones señaladas permiten asumir que los elementos asociados al desarrollo humano se constituyen en factores importantes en las investigaciones y la problemática del desarrollo local y regional. Aspectos relacionados con la cultura, el nivel y la calidad de la educación, la motivación humana y comunitaria, el desarrollo de capacidades de comunicación y liderazgo, el nivel de participación en la toma de decisiones y el grado de construcción de capital social, se asocian con fuerza y de manera simultánea con diversas dimensiones de la vida humana, lo que permite señalar que las investigaciones abordan múltiples dimensiones sociales en las que lo político y cultural aparece integrado y lo económico está implícito, sin que este aspecto sea tratado con una particularidad específica.

Es posible decir entonces, que las investigaciones, le han dado énfasis al estudio de los elementos que promueven de manera directa o indirecta el desarrollo humano, adquiriendo adicionalmente, una relevancia significativa los procesos asociados a la construcción de capital social, concepto que se relaciona con temas como la confianza, la asociatividad y la construcción de redes sociales, aspectos que presuponen formas de despliegue de las potencialidades del ser humano.

El establecimiento de las relaciones referidas fue abordado, en muchos de los casos, a través de la experiencia de comunidades vulnerables localizadas en diversos municipios de la geografía nacional, lo cual resalta la importancia de los factores endógenos en las investigaciones. En otras palabras, las relaciones conceptuales fueron verificadas a través de exploraciones realizadas sobre las experiencias de comunidades, con características específicas dadas, que comparten un espacio socioeconómico común, lo cual se evidencia en el hecho que 17 estudios sistematizan la vivencia de un grupo humano específico. Adicionalmente, estos grupos, generalmente, tienen una participación activa en las diversas etapas del proceso de investigación.

El Cuadro 2 muestra, igualmente, que los problemas de investigación dirigidos a realizar evaluaciones sobre la aplicación de instrumentos, estrategias o metodologías tienen una participación de importancia relativa, ya que en sólo seis casos los trabajos corresponden a esta categoría. Cuatro de estos seis casos se centraron en la evaluación de una medida de política económica o de una estrategia, en el contexto de una comunidad, municipio o grupo poblacional específico, lo cual fue impulsado a través de la administración pública municipal o alguna agencia del orden nacional o internacional. Aunque estos estudios inicialmente se orientaron hacia la búsqueda de la determinación del impacto de la política o estrategia mediante el análisis de variables cuantitativas como el nivel de empleo, la productividad, o indicadores de distribución del ingreso, adicionalmente tienen en cuenta la percepción de la comunidad, con lo cual esta termina involucrándose activamente en la evaluación de la medida de tal manera que las conclusiones rebasan el análisis cuantitativo. 
Respecto a los cinco casos orientados hacia la comprobación de hipótesis, se observa que estas fueron planteadas en términos de la verificación de algunas de las relaciones conceptuales mencionadas al inicio de este aparte. A título de ejemplo, uno de los trabajos explora la hipótesis de que la política estatal y los modelos de desarrollo económico aplicados en Colombia en el último siglo, han reforzado los factores negativos que afectan a la población rural en Colombia (categoría que relaciona "Conflicto, territorio y desarrollo regional”), inhibiendo su posibilidad de alcanzar una mejor calidad de vida. El planteamiento de una relación conceptual lleva implícita la verificación de la hipótesis respectiva.

\section{Tendencias teóricas sobre el Desarrollo y el DEL}

En el acápite anterior se señaló que en los problemas formulados en las investigaciones DEL en Colombia, se identifican elementos asociados con conceptos como desarrollo humano, cultura, capital social, acción colectiva, participación comunitaria, equidad, pobreza y multidimensionalidad.

Los hallazgos, permiten establecer las siguientes categorías para agrupar los elementos conceptuales sobre el tema del Desarrollo Local:

- Desarrollo humano (el hombre como medio y fin) sin referentes específicos sobre el $D E L$.

- Escenario político del desarrollo: nivel de participación en la toma de decisiones y capacidad de autodeterminación de la comunidad.

- Desarrollo como proceso complejo, sistémico o estructural, integrador de una multidimensionalidad (lo económico, lo social, lo cultural, lo político, etc.).

- Desarrollo basado fundamentalmente en variables económicas (elevación del nivel de empleo, la inversión, el consumo, el ingreso o el nivel de producción local; mejoramiento del coeficiente de Gini o de la línea de pobreza).
- Desarrollo basado en las ventajas económicas comparativas regionales.

- Desarrollo enfocado en la promoción de autonomía política y fortalecimiento de los gobiernos locales (descentralización).

- Desarrollo basado en la construcción de capital social.

La clasificación evidencia que la visión del desarrollo local relacionada fundamentalmente con elementos o variables de naturaleza económica en que se enfaticen aspectos cuantitativos, no tiene presencia significativa en las investigaciones. Esta apreciación se ratifica en la categorización teórica presentada en el Cuadro 3. Al identificar los elementos comunes en las categorías establecidas anteriormente, se pueden reconocer cuatro tendencias:

- El desarrollo como un proceso complejo, sistémico o multidimensional. Esta visión incluye los enfoques que abordan al desarrollo a través de la unión y sinergia de elementos de naturaleza política (democratización y participación), cultural (comportamientos, actitudes y valores) y económica y social en sentido general.

- El desarrollo bajo las concepciones del desarrollo humano de MaxNeef (1996) y Sen (1997), la cual involucra la presencia de instancias éticas. En este caso se establece una importante relación de esta orientación con la anterior, dado que el desarrollo humano implica la conexión entre varias dimensiones (cultura, política, economía, ecología, educación, autonomía, libertad) al entenderse al hombre y a la sociedad humana como entes complejos. En esta concepción resulta importante el concepto de capital social y el papel de las instituciones.

- El desarrollo como un proceso de naturaleza esencialmente política, enfoque que también se relaciona de diversas formas con los dos primeros 
dado que en éstos también toman presencia de manera implícita variados elementos de carácter político.

- El desarrollo como un proceso relacionado fundamentalmente con variables exclusivamente eco- nómicas y que implican medición: PIB, empleo, inversión, producción, consumo, ingreso, productividad, costos económicos de oportunidad etc.

El Cuadro 4 describe el resultado de las orientaciones o enfoques conceptuales encontrados.

Cuadro 4. Aspectos conceptuales sobre el desarrollo en general

\begin{tabular}{|l|l|c|}
\hline \multicolumn{1}{|c|}{$\begin{array}{c}\text { Orientaciones teóricas y conceptuales } \\
\text { sobre el desarrollo }\end{array}$} & $\begin{array}{c}\text { Número de } \\
\text { investigaciones }\end{array}$ \\
\hline $\begin{array}{l}\text { Desarrollo como complejidad } \\
\text { Desarrollo humano (el hombre como medio y } \\
\text { fin del desarrollo) }\end{array}$ & 7 \\
\hline $\begin{array}{l}\text { Desarrollo como proceso esencialmente } \\
\text { económico (visión económica tradicional) }\end{array}$ & 3 \\
\hline $\begin{array}{l}\text { Desarrollo como proceso esencialmente } \\
\text { político }\end{array}$ & 3 \\
\hline Sin conceptualización explícita & 2 \\
\hline
\end{tabular}

Fuente: Anexos 3 “Descripción Productos” y 4 “Síntesis Productos Descritos”.

El análisis de la información contenida en el cuadro anterior permite inferir que las investigaciones en lugar de abordar al desarrollo como un tema en donde los elementos económicos son los más importantes, plantean, en general, una visión integradora y holística, posibilitando que resulte más preciso hablar de 'Desarrollo Local' que de 'Desarrollo Económico Local' y en la cual el ser humano, y la organización social por él establecida, son abordados como elementos complejos.

En este escenario, la participación comunitaria se hace esencial, razón por la cual en los procesos de desarrollo el análisis de las instancias políticas es fundamental y sugiere que la construcción de capital social se interpreta en los procesos estudiados, más como una estrategia de carácter político, que una acción dirigida a facilitar el funcionamiento de los mercados, mediante la eliminación o suavización de sus distorsiones e imperfecciones como presupuesto para resolver los problemas del desarrollo, como ha sido planteado en algunas tendencias de la literatura del neoinstitucionalismo (Valdivieso, 2006).

Las tendencias derivadas en los párrafos anteriores, se hacen evidentes cuando se trata de identificar, implícita o explícitamente, la presencia de conceptualizaciones o enfoques que privilegien la dimensión económica en los temas sobre DEL, puesto que lo económico aparece permeado por las otras dimensiones de la sociedad (política, ética, cultural, histórica, etc.). En 22 de las 29 investigaciones revisadas se presenta una definición o delimitación explícita sobre el concepto de Desarrollo Económico Local sin privilegiarse alguna dimensión (Cuadro 1) económica específica y, cuando ésta aparece, la mejoría de lo económico se asume, en algunos casos, como un medio y, en otros, como un objetivo de importancia relativa para mejorar la calidad de vida, pero no como un fin en sí mismo. Solamente en tres se abordan 
aspectos económicos o éstos aparecen como los factores más significativos. En las cuatro restantes, no hay referentes conceptuales alrededor del DEL.

Al interpretar los hallazgos en materia conceptual sobre el $D E L$ se puede advertir:

- La importancia asignada al territorio y al carácter específico de las relaciones sociales de todo tipo que en él se establecen. En la generación de estas relaciones confluyen elementos asociados a diversas dimensiones (económica, política, cultural), las cuales se conectan y refuerzan de tal manera que no resulta posible generalizar que alguna dimensión asuma el papel fundamental. En suma, el desarrollo local se presenta como un proceso que funciona como una estructura, donde todas las partes son relevantes y no es posible entenderlo totalmente desde sólo una de ellas. Por esta razón se valida que la mirada sistémica a los procesos implica la imposibilidad de hacer generalizaciones con respecto al grado de importancia de cada uno de los elementos específicos que conforman el sistema; o, como señala Cuervo (2005) respecto a explicaciones universales sobre el tema.

- El territorio y las relaciones allí generadas son miradas a través de la vivencia concreta de una comunidad, razón por la cual la comprensión de la dinámica de dicha comunidad y de sus relaciones con el entorno juegan un papel fundamental en la investigación sobre $D E L$.

- La concepción de desarrollo apunta significativamente hacia el desarrollo humano, entendiéndose al hombre como un ser complejo, con objetivos múltiples en sus relaciones con un entorno caracterizado por un tejido multidimensional y sistémico de relaciones, por lo que resulta imposible entender los procesos de desarrollo sin abordar integralmente la comprensión de los elementos que determinan, refuerzan, potencian o inhiben la dinámica de las comunidades.

- Se destaca que el papel del ser humano como individuo es fundamental para el logro de una mayor dinámica comunitaria y el proceso de desarrollo resultante. Entonces el desarrollo humano entendido desde el grado de despliegue de sus potencialidades y capacidades, aspectos íntimamente ligados a su vez con el logro de autonomía y libertad, constituye un aspecto crucial para el desempeño de la sociedad en términos de su bienestar y calidad de vida. De aquí que se refuerza la idea que la asociatividad y valores de confianza, reciprocidad y solidaridad (capital social) adquieran un matiz significativamente político y no de simple instrumento económico. Por esto resulta necesario que los procesos de Desarrollo Local reclamen la participación activa de los grupos poblacionales locales.

- El enfoque que privilegia al desarrollo humano asume que las relaciones ubicadas en el contexto de las dimensiones cultural, política, ética, ecológica y antropológica, afectan decisivamente los aspectos asociados a la dimensión económica y que todas en su conjunto conforman un sistema interdependiente. Por lo tanto, el logro de ciertos resultados económicos depende necesariamente de los factores asociados a las demás dimensiones, partiendo de la base de que lo económico solamente juega un papel de relativa importancia en la concepción misma del desarrollo y la calidad de vida.

\section{LOS ENFOQUES METODOLÓGICOS de LAS investigaciones en DEL}

En este aparte el interés se centró en establecer si los estudios realizados asumen enfoques caracterizados por el uso de técnicas asociadas a la investigación de corte cuantitativo o si, en su defecto, los trabajos 
fueron abordados principalmente a través de procesos inherentes a la investigación cualitativa. Adicionalmente, se revisó el proceso de obtención de la información con el fin de evidenciar la preferencia por el uso de fuentes primarias o secundarias en los estudios y, en este último caso, los instrumentos utilizados para la obtención de dicha información.

Los marcos conceptuales que se tuvieron en cuenta para la diferenciación de los enfoques metodológicos son:

Investigación cuantitativa: enfoque basado principalmente en alguno de los siguientes ejercicios: cuantificación de variables; medición del grado o nivel de relación (o correlación) entre elementos o variables; verificación empírica de modelos mediante el uso de técnicas matemáticas; uso fundamental de índices, estadísticas o series numéricas históricas; inferencia estadística mediante el uso de encuestas utilizando técnicas de muestreo; establecimiento de relaciones mediante el uso fundamental de datos numéricos o cuantificables; verificación empírica de hipótesis a través de hechos formulados o expresados numéricamente; y cuantificación del impacto de un fenómeno o medida de política económica.
Investigación cualitativa: proceso de investigación realizado fundamentalmente mediante alguno de los siguientes procesos: el método de Investigación Acción Participación IAP; uso de entrevistas abiertas o semiestructuradas de tipo cualitativo; identificación de grupos focales y aplicación de talleres colectivos; estudios de opinión o percepción sobre aspectos cualitativos; establecimiento de marcos de horizontalidad con las comunidades abordadas (niveles iguales, procesos dialógicos de comunicación y generación de conocimiento); convivencia con la comunidad y registro de la información mediante la elaboración de diarios de campo; y diseño participativo de la investigación.

Investigación documentaria: se incluyó esta categoría dada la importancia relativa de este tipo de estudios en las investigaciones sobre el DEL en Colombia. Este tipo de investigación se caracteriza por la ausencia de contacto con los miembros de alguna comunidad. La obtención de la información es realizada a través de procesos de revisión de libros, artículos y documentos, lo que implica que los resultados y conclusiones son obtenidos a través de la aplicación de procesos de naturaleza hermenéutica. El Cuadro 5 resume los principales hallazgos sobre este aspecto:

Cuadro 5. Fuentes de información y enfodues metodológicos

\begin{tabular}{|l|l|c|}
\hline \multicolumn{1}{|c|}{ Fuentes } & \multicolumn{1}{|c|}{ Enfoques } & Número de trabajos \\
\hline \multirow{2}{*}{ Fundamentalmente primarias } & Cualitativo & 19 \\
\cline { 2 - 3 } & Cuantitativo & 2 \\
\hline Fundamentalmente secundarias & $\begin{array}{l}\text { Investigaciones esencialmente } \\
\text { documentarias o bibliográficas. }\end{array}$ & 8 \\
\hline \multicolumn{2}{|l}{ TOTAL TRABAJOS } & 29 \\
\hline
\end{tabular}

Fuente: Anexos 3 “Descripción Productos” y 4 “Síntesis Productos Descritos”.

Los resultados de la tabla anterior permiten establecer que la investigación de corte esencialmente cualitativo adquiere un carácter relevante en los estudios. Ese resultado es coherente con la importancia de la dinámica

70 / Álvaro Albán Moreno / Jorge Alberto Rendón Vélez 
participativa característica del proceso y con la naturaleza compleja y multidimensional de los problemas abordados, la cual dirige los estudios en mayor medida al entendimiento de relaciones conceptuales de carácter sistémico que a la cuantificación o determinación del grado de relevancia de variables específicas.

\section{Resultados y conclusiones de las INVESTIGACIONES SOBRE DEL}

En el presente título se ilustra una agrupación de las respuestas encontradas a los problemas e hipótesis formuladas en las investigaciones. Es destacable que ambas categorías de agrupación se relacionan íntimamente.

Sobre el DEL como instrumento, actividad, propuesta de solución a un problema, acción de política, proceso o estrategia de desarrollo, se destacan:

- La importancia que tienen los mecanismos o estrategias dirigidas a fomentar la participación de las comunidades en los procesos de investigación, de decisión presupuestal o políticos y en general de consecución del desarrollo. Aspectos como el potenciamiento de los valores de liderazgo, solidaridad y asociatividad, se validan para la búsqueda de un mayor nivel de autonomía.

- Las posibilidades de encontrar soluciones mejores para las comunidades con dificultades socioeconómicas, asociadas a la construcción de redes sociales, lazos de afectividad y solidaridad, autorreconocimiento y visibilización social, y generación de capital social.

- La educación es reconocida como factor relevante en el $D E L$, en tanto que presupone resignifica el sentido de la participación comunitaria.

- El territorio constituye un elemento contextual, que facilita el establecimiento de diversas clases de conexiones entre las personas (económicas, culturales, etnográficas, sociales, psicológicas, etc.) y donde la comunidad ejercita su derecho a la búsqueda de un mejor nivel de vida, lo que incluye la posibilidad de confrontación de las estructuras económicas y de poder establecidas. El poder sobre el territorio también es fuente de conflicto, marginación y desplazamiento.

- El papel que en el desarrollo de la localidad tienen los nuevos yacimientos de empleo -NYE- (esparcimiento, cultura, deportes, renovación espacio urbano, ampliación de vivienda sectores de bajos ingresos y formación de jóvenes desempleados).

- La planeación participativa, democratización y su relación con el desarrollo local.

- Las posibilidades y dificultades de la relación entre información, planeación del desarrollo y toma de decisiones.

- Los estudios de caso sobre descentralización y DEL.

- La descripción de circuitos económicos, concentración de mercados y condiciones de pobreza de comunidades.

Sobre los aportes teóricos y/o conceptuales se encontró:

- La exploración de las relaciones entre cultura y estructura de la pobreza; y la multidimensionalidad de los conceptos de pobreza, calidad de vida, desarrollo y bienestar.

- El enfoque del desarrollo orientado primordialmente hacia el desarrollo humano en términos del despliegue de potencialidades y capacidades, dirigidos a la búsqueda de una mayor autonomía y libertad. En esta perspectiva, la dimensión política está implícita como aspecto determinante del desarrollo en la localidad.

- El contraste entre descripciones objetivas de realidades sociales, con las valoraciones subjetivas o punto de vista de la comunidad y las inferencias que provoca, sobre relaciones de dominación, participación de lo público, y conocimiento, significado y reconocimiento de la pobreza por parte de la gente que la vive y sufre.

- La relación entre localidad, competitividad y desarrollo. 
- El reconocimiento del origen, tipos de estrategias, instrumentos y acciones de política derivadas; y el reconocimiento de los límites del DEL como teoría y práctica.

- La exploración de nuevos paradigmas mediante el análisis de métodos empíricos y teóricos: economía experimental, teoría de juegos y diagnóstico participativo.

\section{RECONSTRUCCIONES INTERPRETATIVAS Y VALORACIONES}

- Al reconocerse el papel de la participación, la generación de capital social, y las implicaciones complejas del territorio en los resultados y conclusiones de las investigaciones, como elementos consustanciales de los procesos de DEL, ello constituye un hallazgo empírico de la presente investigación, sobre el carácter esencialmente político del Desarrollo Económico Local, en tanto que en él confluyen las relaciones económicas, políticas y culturales de una comunidad.

- Las investigaciones evidencian la idea que los diversos territorios, a pesar de sus diferencias, comparten un elemento en común: facilitan la generación de lazos entre las personas y parecen adquirir fundamentalmente el carácter de espacios de acción política, conectando a los individuos a través de la búsqueda de solución a los conflictos, la vivencia de problemas comunes, los vínculos étnicos y culturales, la necesidad de acceso al uso o posesión de los recursos regionales o locales, entre otros aspectos. Ello le asigna al territorio un papel determinante al Desarrollo Local, en tanto que constituye especificidad y generalidad.

- Los estudios sobre pobreza y calidad de vida asumidos desde la perspectiva del desarrollo local, sugieren la necesidad de involucrar con mayor fuerza la mirada de quienes habitan la pobreza y la marginalidad social y económica. Los pobres construyen la realidad pero no siempre desde sus propios intereses. La comprensión de la comunidad de la realidad social no es autónoma, pues está mediada por un marco institucional, histórico, económico y político inherente al ordenamiento social: hogar, escuela, medios masivos de comunicación, relaciones de poder, etc. Es necesario fortalecer los enfoques investigativos que amplían la mirada sobre las interpretaciones y soluciones de la pobreza, que generalmente han estado centrados en la posibilidad de obtención de un ingreso mínimo o de satisfacción de necesidades básicas, lo que propicia una aceptación per se, que nivela por lo bajo las posibilidades de bienestar. En esta perspectiva, quedan por fuera temas como la libertad, los derechos, las capacidades y las oportunidades. Congruente con lo anterior, se valida la crítica que ha sido efectuada a la investigación en ciencias sociales, cuando ésta sólo aborda la dimensión cuantitativa de la realidad, dejando por fuera las relaciones intersubjetivas, situación que ha sido calificada, como un "sesgo metodológico” (Bonilla-Castro y Rodríguez, 2005).

- La investigación universitaria en Desarrollo Local en Colombia, abarca cuatro niveles de actuación, a saber: Político-Administrativo: participación, democratización, descentralización y transformaciones sociales; Cultural-Institucional: redes sociales y capital social; Económico-Productivo: competitividad, mercados, uso eficiente de los recursos, modelos de localización. Geográfico-Ambiental; uso y explotación de recursos naturales.

- En suma, este trabajo de investigación, permite concluir, para el caso colombiano, sobre las evidencias respecto a la naturaleza de estructura del concepto y práctica del Desarrollo Local y sobre su carácter multidimensional y transdimensional.

\section{Conclusiones y Recomendaciones}

¿Qué esfuerzo son necesarios para avanzar en los procesos investigativos universitarios en DEL? 
- La investigación universitaria en $D E L$ requiere ahondar esfuerzos en el diálogo entre la academia y la comunidad. Este estudio permite señalar que se han hecho trabajos investigativos desde las universidades de corte participativo en diversas localidades en Colombia, pero se hace necesario involucrar un mayor número de trabajos a lo largo de la geografía nacional con el fin de lograr resultados que permitan avanzar en el desarrollo de un concepto en donde no son posibles las generalizaciones. La universidad colombiana debe asumir su papel no solamente como agente generador de conocimiento, sino también de reflexión con sentido crítico y transformación, para lo cual el diálogo con la comunidad se plantea como una condición necesaria en la construcción de escenarios para el desarrollo de las prácticas, metodologías y acciones encaminadas hacia el mejoramiento de la calidad de vida y el bienestar de la población en la perspectiva local.

¿Qué tan cercana al concepto de Desarrollo Económico Local definido en el presente trabajo, están las investigaciones universitarias?

- La tendencia de la investigación universitaria de los grupos registrados en Colciencias alrededor del tema del Desarrollo Económico Local, se ha orientado en mayor medida hacia la evaluación y el desarrollo de estrategias, prácticas y metodologías asociadas con el tema (es decir, al proceso en sí mismo) y no hacia la posible construcción o afinamiento de su cuerpo teórico y conceptual. Por lo tanto, todavía hay mucho por hacer con respecto a la fundamentación teórica del concepto de Desarrollo Económico Local y su contrastacción con la realidad, situación que dificulta la identificación de los procesos, estrategias y políticas asociadas con el $D E L$ lo que puede conllevar a generar efectos y resultados de muy corto plazo, tanto en los procesos de investigación como de intervención en las comunidades. Se requiere un mayor avance de la investigación en este sentido, lo que seguramente permitirá un mejor impacto de los estudios y trabajos. Hay algunas excepciones, como la del Centro de Investigaciones sobre el Desarrollo Económico Regional CIDER de la Universidad de los Andes, donde se ha realizado un importante trabajo de reflexión teórica y conceptual.

¿Qué sugerencia se hacen respecto al futuro de las investigaciones universitarias en DEL? ¿Debería indagarse con mayor profundidad los efectos de estos procesos en el desarrollo de las comunidades objeto de las investigaciones?

- Es recomendable que la investigación universitaria sobre DEL genere metodologías y prácticas que permitan que los procesos realizados tengan la continuidad necesaria y sean objeto de una evaluación subsiguiente, de tal manera que sea posible verificar su impacto en las comunidades en donde se han realizado. Por lo tanto se requiere complementar las investigaciones con la creación de observatorios socioeconómicos y sistemas de monitoreo con el fin de evaluar el resultado y contribución tanto de los estudios realizados como de las dinámicas sociales y económicas resultantes de estos. Es necesario evaluar hasta qué punto los procesos investigativos han tenido impacto en la generación de políticas y cuáles factores dinamizan o inhiben su sostenibilidad.

Finalmente, este trabajo permite plantear a sus autores, que la asunción del Desarrollo Económico Local o el Desarrollo Local, como un concepto integrador de todas las dimensiones sociales locales (economía, política, cultura, instituciones, historia), en el que la particularidad impide las generalizaciones teóricas, implica avanzar en la propuesta interdisciplinaria tal como su naturaleza de estructura lo requiere. 


\section{Bibliografía}

Albán, A. y Zapata, H. La información socioeconómica en contextos de desarrollo local. El caso Sevilla Valle. Cali: Universidad Libre Seccional Cali, 2005.

Abello, R. y otros. "Redes sociales: un mecanismo de supervivencia en sectores de pobreza". Revista Investigación y Desarrollo 2. 4 (1995): 73 - 89.

Aguado, L. "Desarrollo Económico Local y Nuevos Yacimientos del Empleo para Cali”. Revista Economía, Gestión y Desarrollo. 2 (2004): 101 - 122.

Alburquerque, F. "El enfoque del Desarrollo Económico Local”. Cuadernos DEL I. Buenos Aires, 2004.

Amaris, M. et al. "Potencialidades para el desarrollo comunitario. Un estudio de caso en familias de la Costa Norte colombiana”. Revista Investigación y Desarrollo 8. 4 (1996): 9 - 28.

Cárdenas, J. et al. "Métodos experimentales y participativos para el análisis de la acción colectiva y la cooperación en el uso de recursos naturales por parte de comunidades rurales". Cuadernos de desarrollo rural. 50 (2003): 63 - 97.

Cardona, S. "Educación y Desarrollo Local”. Educación y Sociedad. Manizales: Universidad Católica de Manizales, 2000.

Castañeda, H. "Estrategias de Desarrollo Económico Local y participación comunitaria: el caso de desmarginalización en Bogotá". Revista Economía y Desarrollo 4.1 (2005): 113 - 143.

Cavestany, F. Desarrollo desde el territorio. A propósito del Desarrollo Local. Documento REDEL. 2000.

Correa, A. "Una nueva cultura política. Un caso de presupuesto participativo". Revista Investigación y Desarrollo 8. (1998): 12 - 39.
Cuervo, L. "Desarrollo económico local: leyendas y realidades". Revista Territorios. Asociación Colombiana de Investigadores Urbano. (2005): 9 - 24.

Cuervo, L. et al. Veinte planes de desarrollo local. Capacitación, estructuración y seguimiento. Elementos de gestión participativa. Santafé de Bogotá: Centro Interdisciplinario de Estudios Regionales. Cider, Universidad de los Andes, 2002.

Bonilla, E. et al. Más allá del dilema de los métodos. La investigación en ciencias sociales. Bogotá: Norma, 2005.

Gallo, L. "Procesos de desarrollo comunitario asociados a los diferentes estilos de liderazgo en las comunidades negras de la ciudad de Barranquilla”. Revista Investigación y Desarrollo 6. (1997): $97-104$.

García, L. "Desarrollo implicad". Revista de investigaciones de la Universidad Católica de Manizales. 6 (2005): $9-20$.

Gaviria, M. "La problemática del desarrollo colombiano”. Revista Páginas 59. (2001): 15 - 26.

Gil, A. "Aproximación conceptual a los términos desarrollo, bienestar y calidad de vida”. Revista Páginas 65. (2003): 45 - 68.

Jiménez, S. et al. Desarrollo Local y calidad de vida en Cartagena. Caracterización de los barios El Central, El Milagro y San José de los Campanos. Serie de documentos No.1 Cartagena: Universidad de San Buenaventura, 2003.

Lacera, R. El turismo como estrategia de Desarrollo Económico Local. Memoria de grado para optar el título de economista. Universidad de los Andes. 2002.

Maldonado, A. La experiencia de desarrollo local en el municipio de Pensilvania Caldas. Proyecto CEPAL - GTZ. Santiago de Chile. 2000. 
Maradiago, C. et al. "Potencialidades del desarrollo humano respecto a su calidad de vida en un asentamiento de la Costa Caribe colombiana”. Revista Investigación y Desarrollo 9. (1999): 9- 28.

Maradiago, C. et al. "Satisfactores de necesidades humanas asociadas a las redes sociales de jóvenes escolarizados en la ciudad de Barranquilla”. Revista Investigación y Desarrollo 6. (1997): 1 16.

Marulanda, D. Un modelo de gestión pública local del desarrollo con gobernabilidad. Medellín: Gobernación de Antioquia, 2004.

MaxNeef. M. Desarrollo a escala humana: una opción para el futuro. Santiago de Chile: Nordan Comunidad, 1986.

Narváez, L. Circuitos económicos y desarrollo local. Caso de estudio población de Hojas Blancas. Municipio de Francisco Pizarro. Salahonda. Pacífico sur colombiano. Santafé de Bogotá: Centro Interdisciplinario de Estudios Regionales Cider, Universidad de los Andes, 2003.

Palacios, J. et al. "El capital social como apoyo a la superación de problemas en la población desplazada por la violencia en Colombia”. Revista Investigación y Desarrollo 9. 2 (2006): 514 - 535.

Pallares, O. "Análisis de la cultura de la pobreza". Revista Investigación y Desarrollo 4. (1996): 90 113.
Parada, J. “Ciudad y apertura económica”. Revista Investigación y Desarrollo 7. (1999): 29 - 40.

Pérez, M. "La conformación territorial en Colombia: entre el conflicto, el desarrollo y el destierro”. Revista Cuadernos de Desarrollo Rural 51. (2004): $61-90$.

Rendón, J. \& Gutiérrez, E. Competitividad y Localidad: una opción para el desarrollo. Tesis de maestría. Universidad Pontificia Bolivariana, Medellín. 2004.

Sen, A. Sobre ética y economía. Madrid: Alianza Editorial, 1997.

Tello, M. Las teorías del desarrollo económico local y la teoría y práctica del proceso de descentralización en los países en desarrollo. Departamento de Economía y CENTRUN CATÓLICA. Consorcio de Investigaciones Económicas y Sociales (CIES) Florida, 2006. http:/www.pucp.edu.pe/economia/ pdf/DDD247.pdf

Toro, D. "Competitividad y localización de empresas de Cartagena frente a la evidencia colombiana en los noventa”. Revista Serie de estudios sobre competitividad en Cartagena. 2. (2002): 3 - 27.

Valdivieso, S. “¿Nuevos aires en la teoría del desarrollo?". Revista Latinoamericana de Economía. Problemas de desarrollo 37. 144 (2006): 11 - 32.

Vélez, Y. "Usos de la información para la toma de decisiones en procesos de desarrollo local”. Revista Investigación y Desarrollo 8. (1998). 